\title{
Robust Controller Design for Timed Event Graphs in Dioids
}

\author{
Mehdi Lhommeau, Laurent Hardouin, Bertrand Cottenceau, Luc Jaulin
}

\begin{abstract}
This paper deals with feedback controller synthesis for timed event graphs in dioids, where the number of initial tokens and time delays are only known to belong to intervals. The synthesis presented here is mainly based on dioid, interval analysis and residuation theory.

keyword

Discrete-event systems ; Timed event graphs ; Dioid ; Interval analysis ; Residuation theory ; Feedback synthesis
\end{abstract}

\section{INTRODUCTION}

Discrete Event Systems (DES) appear in many applications in manufacturing systems [1], computer and communication systems [4] and are often described by the Petri Net formalism. Timed-Event Graphs (TEG) are Timed Petri Nets in which all places have single upstream and single downstream transitions and appropriately model DES characterized by delay and synchronization phenomena. TEG can be This specification means, not more than two tokens can input in the TEG at the same moment.described by linear equations in the dioid algebra [2], [5] and this fact has permitted many important achievements on the control of DES modelled by TEG [5], [6], [10]. TEG control problems are usually stated in a Just-intime context. The design goal is to achieve some performance while minimizing internal stocks. In [6] linear closed-loop controllers synthesis are given in a model matching objective, i.e., a given reference model describes the desired performance limits, then the goal is to compute a feedback controller in order to obtain a closed-loop behavior as close as possible to the reference model and to delay as much as possible the inputs in the system.

This paper aims at designing robust feedback controller when the system includes some parametric uncertainties which can be described by intervals. Intervals allow to describe TEG with number of tokens and/or time delays, which are assumed to vary between known bounds. Assuming that there exists a lower and an upper bound to a specification set, the synthesis yields a controller set which guarantees that the closed loop system behavior is both greater than the lower bound of the specification set and lower than the upper bound of this same set ${ }^{1}$. Controller synthesis is obtained by considering residuation theory which allows the inversion of mapping defined over ordered sets, and interval analysis which is known to be efficient to characterize set of robust controllers in a

\footnotetext{
Laboratoire d'Ingénierie des Systèmes Automatisés, 62, avenue NotreDame du lac, 49000 ANGERS, FRANCE, Tel: (33) 2413657 33, Fax: (33) 2 413657 35. E-mail: [mehdi.lhommeau,bertrand.cottenceau, laurent.hardouin, luc.jaulin]@istia.univ-angers.fr

${ }^{1}$ From TEG point of view it is a set of robust controller which ensures that the controlled system is both slower than a reference model (described as a TEG) and faster than another one.
}

guaranteed way [8].

The next Section introduces algebraic tools on dioid and residuation theory. Section 3 is devoted to a dioid of interval $\mathrm{I}(\mathcal{D})$ and mapping inversion over $\mathrm{I}(\mathcal{D})$. The problem of robust controller synthesis when the system includes interval parametric uncertainties is stated and solved in Sections 5. Section 6 presents an illustrative example.

\section{DIOIDS AND RESIDUATION}

A dioid $\mathcal{D}$ is a set endowed with two internal operations denoted $\oplus$ (addition) and $\otimes$ (multiplication), both associative and both having neutral elements denoted $\varepsilon$ and $e$ respectively, such that $\oplus$ is also commutative and idempotent (i.e. $a \oplus a=$ $a)$. The $\otimes$ operation is distributive with respect to $\oplus$, and $\varepsilon$ is absorbing for the product (i.e. $\varepsilon \otimes a=a \otimes \varepsilon=\varepsilon, \forall a$ ). When $\otimes$ is commutative, the dioid is said to be commutative. The symbol $\otimes$ is often omitted.

Dioids can be endowed with a natural order : $a \succeq b$ iff $a=a \oplus b$. Then they become sup-semilattices and $a \oplus b$ is the least upper bound of $a$ and $b$. A dioid is complete if sums of infinite number of terms are always defined, and if multiplication distributes over infinite sums too. In particular, the sum of all elements of the dioid is defined and denoted $\top$ (for 'top'). A complete dioid (sup-semilattice) becomes a lattice by constructing the greatest lower bound of $a$ and $b$, denoted $a \wedge b$, as the least upper bound of the (nonempty) subset of all elements which are less than $a$ and $b$ (see [2, $\S 4])$.

Example $1\left(\overline{\mathbb{Z}}_{\max }\right.$ dioid $)$ : The set $\overline{\mathbb{Z}}=\mathbb{Z} \cup\{-\infty,+\infty\}$ endowed with the max operator as sum and the classical sum + as product is a complete dioid, usually denoted by $\overline{\mathbb{Z}}_{\text {max }}$, of which $\varepsilon=-\infty$ and $e=0$.

Theorem 1: Over a complete dioid $\mathcal{D}$, the implicit equation $x=a x \oplus b$ admits $x=a^{*} b$ as least solution, where $a^{*}=$ $\bigoplus_{i \in \mathbb{N}} a^{i}$ (Kleene star operator) with $a^{0}=e$.

Theorem 2 ([6]): Let $\mathcal{D}$ be a complete dioid and $a, b \in \mathcal{D}$.

$$
\begin{aligned}
a(b a)^{*} & =(a b)^{*} a, \\
\left(a^{*}\right)^{*} & =a^{*}
\end{aligned}
$$

\section{A. Residuation theory}

The residuation theory provides, under some assumptions, optimal solutions to inequalities such as $f(x) \preceq b$ where $f$ is an isotone mapping ( $f$ s.t. $a \preceq b \Rightarrow f(a) \preceq f(b)$ ) defined over ordered sets. Some theoretical results are summarized below. Basic references are [3] and [2, §4.4.2].

Definition 1 (Residual and residuated mapping): An isotone mapping $f: \mathcal{D} \rightarrow \mathcal{E}$, where $\mathcal{D}$ and $\mathcal{E}$ are ordered 
sets, is a residuated mapping if for all $y \in \mathcal{E}$, the least upper bound of the subset $\{x \mid f(x) \preceq y\}$ exists and belongs to this subset. It is then denoted $f^{\sharp}(y)$. Mapping $f^{\sharp}$ is called the residual of $f$. When $f$ is residuated, $f^{\sharp}$ is the unique isotone mapping such that

$$
f \circ f^{\sharp} \preceq \operatorname{Id}_{\mathcal{E}} \quad \text { and } \quad f^{\sharp} \circ f \succeq \operatorname{Id}_{\mathcal{D}},
$$

where Id is the identity mapping respectively on $\mathcal{D}$ and $\mathcal{E}$.

Property 1: Let $f: \mathcal{D} \rightarrow \mathcal{E}$ be a residuated mapping, then

$$
y \in f(\mathcal{D}) \Leftrightarrow f\left(f^{\sharp}(y)\right)=y .
$$

Theorem 3 ([2, §4.4.2]): Consider the mapping $f: \mathcal{E} \rightarrow$ $\mathcal{F}$ where $\mathcal{E}$ and $\mathcal{F}$ are complete dioids of which the bottom elements are, respectively, denoted by $\varepsilon_{\mathcal{E}}$ and $\varepsilon_{\mathcal{F}}$. Then, $f$ is residuated iff $f\left(\varepsilon_{\mathcal{E}}\right)=\varepsilon_{\mathcal{F}}$ and $f\left(\bigoplus_{x \in \mathcal{G}} x\right)=\bigoplus_{x \in \mathcal{G}} f(x)$ for each $\mathcal{G} \subseteq \mathcal{E}$ (i.e $f$ is lower-semicontinuous abbreviated l.s.c.).

Corollary 1: The mappings $L_{a}: x \mapsto a x$ and $R_{a}: x \mapsto x a$ defined over a complete dioid $\mathcal{D}$ are both residuated. ${ }^{2}$ Their residuals are usually denoted, respectively, $L_{a}^{\sharp}(x)=a \oint x$ and $R_{a}^{\sharp}(x)=x \phi a$ in $(\max ,+)$ literature. $^{3}$

Theorem 4 ([2, §4.4.4]): The mappings $x \mapsto a \oint x$ and $x \mapsto$ $x \phi a$ verify the following properties :

$$
\begin{array}{rc}
(a b) \phi x=b \phi(a \phi x) & x \phi(b a)=(x \phi a) \phi b, \\
a^{*} x=a^{*} \phi\left(a^{*} x\right) & x a^{*}=\left(x a^{*}\right) \phi a^{*}, \\
a \phi(x \wedge y)=a \phi x \wedge a \phi y & (x \wedge y) \phi a=x \phi a \wedge y \phi a .
\end{array}
$$

\section{DiOID AND INTERVAL MATHEMATICS}

Interval mathematics was pioneered by Ramon E. Moore as a tool for bounding rounding and truncation errors in computer programs. Since then, interval mathematics had been developed into a general methodology for investigating numerical uncertainty in numerous problems and algorithms, and is a powerful numerical tool for calculating guaranteed bounds on functions using computers.

In [9] the problem of interval mathematics in dioids is addressed. The authors give a weak interval extensions of dioids and show that idempotent interval mathematics appears to be remarkably simpler than its traditional analog. Below, we state that residuated theory has a natural extension in dioid of intervals.

\section{A. Interval arithmetic in dioid}

A (closed) interval in dioid $\mathcal{D}$ is a set of the form

$$
\mathbf{x}=[\underline{x}, \bar{x}]=\{t \in \mathcal{D} \mid \underline{x} \preceq t \preceq \bar{x}\}
$$

where $(\underline{x}, \bar{x}) \in \mathcal{D}^{2}, \underline{x}$ (respectively, $\bar{x}$ ) is said to be lower (respectively, upper) bound of the interval x. In [9] the authors define from a dioid $\mathcal{D}$ a dioid of interval, denoted $\mathrm{I}(\mathcal{D})$, endowed with two coordinate-wise algebraic operations :

$$
\begin{aligned}
& \mathbf{x} \oplus \mathbf{y}=[\underline{x} \oplus \underline{y}, \bar{x} \oplus \bar{y}] \\
& \mathbf{x} \bar{\otimes} \mathbf{y}=[\underline{x} \otimes \underline{y}, \bar{x} \otimes \bar{y}]
\end{aligned}
$$

\footnotetext{
${ }^{2}$ This property concerns as well a matrix dioid product, for instance $X \mapsto$ $A X$ where $A, X \in \mathcal{D}^{n \times n}$. See [2] for the computation of $A \phi B$ and $B \phi A$.

${ }^{3} a \phi b$ is the greatest solution of $a x \preceq b$.
}

where the interval $\varepsilon=[\varepsilon, \varepsilon]$ (respectively, $\mathbf{e}=[e, e]$ ) is zero (respectively, unit) element of $\mathrm{I}(\mathcal{D})$.

Since $\underline{x} \oplus y \preceq \bar{x} \oplus \bar{y}$ and $\underline{x} \otimes y \preceq \bar{x} \otimes \bar{y}$ whenever $\underline{x} \preceq \bar{x}$ and $\underline{y} \preceq \bar{y}$, then $\mathrm{I}(\mathcal{D})$ is closed with respect to the operations $\bar{\oplus}, \bar{\otimes}$. Dioid $\mathrm{I}(\mathcal{D})$ can be completed by considering the following definition.

Definition 2: Let $\left\{\mathbf{x}_{\alpha}\right\}$ be an infinite subset of $\mathrm{I}(\mathcal{D})$, the infinite sum of elements of this subset is :

$$
\bigoplus_{\alpha} \mathbf{x}_{\alpha}=\left[\bigoplus_{\alpha} \underline{x}_{\alpha}, \bigoplus_{\alpha} \bar{x}_{\alpha}\right]
$$

Dioid $\mathrm{I}(\mathcal{D})$ can be endowed with a natural (partial) order :

$$
\mathbf{a} \succeq_{\mathrm{I}(\mathcal{D})} \mathbf{b} \Leftrightarrow \mathbf{a}=\mathbf{a} \bar{\oplus} \mathbf{b} \Leftrightarrow \bar{a} \succeq_{\mathcal{D}} \bar{b} \text { and } \underline{a} \succeq_{\mathcal{D}} \underline{b}
$$

An interval for which $\underline{x}=\bar{x}$ is called degenerate. Degenerate intervals allow to represent numbers without uncertainty. In this case we identify $\mathrm{x}$ with its element by writing $\mathrm{x} \equiv x$.

Remark 1: Note that if $\mathbf{x}$ and $\mathbf{y}$ are intervals in $\mathrm{I}(\mathcal{D})$, then

$$
\mathbf{x} \subset \mathbf{y} \Leftrightarrow \underline{y} \preceq \underline{x} \preceq \bar{x} \preceq \bar{y}
$$

In particular, $\mathbf{x}=\mathbf{y}$ iff $\underline{x}=\underline{y}$ and $\bar{x}=\bar{y}$.

Remark 2: $\mathrm{I}(\mathcal{D})$ being closed with respect to the operations $\bar{\oplus}, \bar{\otimes}$, the Kleene star operator admits a natural extension, thus

$$
\mathrm{x}^{*}=\bigoplus_{i \in \mathbb{N}} \mathrm{x}^{i}=\left[\bigoplus_{i \in \mathbb{N}} \underline{x}^{*}, \bigoplus_{i \in \mathbb{N}} \bar{x}^{*}\right]=\left[\underline{x}^{*}, \bar{x}^{*}\right]
$$

with $\mathbf{x}^{0}=\mathbf{e}$.

\section{B. Residuation of interval linear inequations}

Proposition 1: Mapping $L_{\mathbf{a}}: \mathrm{I}(\mathcal{D}) \rightarrow \mathrm{I}(\mathcal{D}), \mathbf{x} \mapsto \mathbf{a} \bar{\otimes} \mathbf{x}$ is residuated. Its residual is equal to

$$
L_{\mathbf{a}}^{\sharp}(\mathbf{b})=\mathbf{a} \phi \mathbf{b}=[\underline{a} \oint \underline{b} \wedge \bar{a} \phi \bar{b}, \bar{a} \phi \bar{b}]
$$

Proof: Observe that $L_{\mathbf{a}}$ is 1.s.c., i.e., $L_{\mathbf{a}}\left(\bar{\bigoplus}_{\mathbf{x} \in \mathbf{X}} \mathbf{x}\right)=\bar{\bigoplus}_{\mathbf{x} \in \mathbf{X}} L_{\mathbf{a}}(\mathbf{x})$, (for every subset $\mathbf{X}$ of $\mathrm{I}(\mathcal{D})$ ), moreover $L_{\mathbf{a}}(\varepsilon)=L_{\mathbf{a}}([\varepsilon, \varepsilon])=[\underline{a} \varepsilon, \bar{a} \varepsilon]=[\varepsilon, \varepsilon]=\varepsilon$. Then $L_{\mathbf{a}}$ is residuated (see Theorem 3 ).

It remains to obtain the analytical expression of $L_{\mathbf{a}}^{\sharp}(\mathbf{b})=\mathbf{a} \oint \mathbf{b}$. It is the greatest $\mathbf{x}$ solution of $\mathbf{a x} \preceq \mathbf{b}$, this inequality can be written as follows :

$$
\begin{gathered}
{[\underline{a}, \bar{a}] \bar{\otimes}[\underline{x}, \bar{x}] \preceq_{\mathrm{I}(\mathcal{D})}[\underline{b}, \bar{b}]} \\
\Leftrightarrow \underline{a} \otimes \underline{x} \preceq_{\mathcal{D}} \underline{b} \text { and } \bar{a} \otimes \bar{x} \preceq_{\mathcal{D}} \bar{b} \text { and } \underline{x} \preceq_{\mathcal{D}} \bar{x}
\end{gathered}
$$

The last inequality ensures that $\mathbf{x}$ is not empty. Theorem 11 leads to

$$
\underline{x} \preceq_{\mathcal{D}} \underline{a} \phi \underline{b} \quad \text { and } \quad \bar{x} \preceq_{\mathcal{D}} \bar{a} \phi \bar{b} \quad \text { and } \quad \underline{x} \preceq_{\mathcal{D}} \bar{x}
$$

thus the greatest interval is obviously given by :

$$
\mathbf{x}=[\underline{x}, \bar{x}] \preceq_{\mathrm{I}(\mathcal{D})}[\underline{a} \oint \underline{b} \wedge \bar{a} \phi \bar{b}, \bar{a} \phi \bar{b}]
$$

Remark 3: We would show in the same manner that mapping $R_{\mathbf{a}}: \mathrm{I}(\mathcal{D}) \rightarrow \mathrm{I}(\mathcal{D}), \mathbf{x} \mapsto \mathbf{x} \otimes \mathbf{a}$ is residuated. 


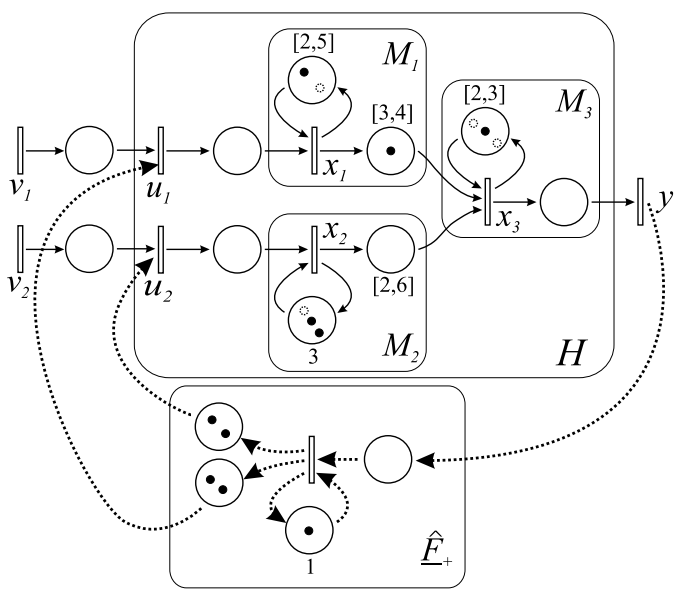

Fig. 1. A uncertain TEG with a controller (bold dotted lines)

\section{INTERVAL ARITHMETIC AND TIMED EVENT GRAPHS}

It is well known that the behavior of a TEG can be expressed by linear state equations over some dioids, e.g., over dioid of formal power series with coefficients in $\overline{\mathbb{Z}}_{\max }$ and exponents in $\mathbb{Z}$ namely $\overline{\mathbb{Z}}_{\max } \llbracket \gamma \rrbracket$.

$$
\begin{aligned}
& X=A X \oplus B U \\
& Y=C X
\end{aligned}
$$

Where $X \in\left(\overline{\mathbb{Z}}_{\max } \llbracket \gamma \rrbracket\right)^{n}$ represents the internal transitions behavior, $U \in\left(\overline{\mathbb{Z}}_{\max } \llbracket \gamma \rrbracket\right)^{p}$ represents the input transitions behavior, and $Y \in\left(\overline{\mathbb{Z}}_{\max } \llbracket \gamma \rrbracket\right)^{q}$ represents the output transitions behavior, and $A \in\left(\overline{\mathbb{Z}}_{\max } \llbracket \gamma \rrbracket\right)^{n \times n}, B \in\left(\overline{\mathbb{Z}}_{\max } \llbracket \gamma \rrbracket\right)^{n \times p}$ and $C \in\left(\overline{\mathbb{Z}}_{\max } \llbracket \gamma \rrbracket\right)^{q \times n}$ represent the link between transitions. We refer the reader to [5] for a complete presentation.

The class of uncertain systems, which will be considered, are TEG where the number of tokens and time delays are only known to belong to intervals. Therefore uncertainties can be described by intervals with known lower and upper bounds and the matrices of Equations (9) and (10) are such that $A \in \mathbf{A} \in \mathrm{I}\left(\overline{\mathbb{Z}}_{\max } \llbracket \gamma \rrbracket\right)^{n \times n}, B \in \mathbf{B} \in \mathrm{I}\left(\overline{\mathbb{Z}}_{\max } \llbracket \gamma \rrbracket\right)^{n \times p}$ and $C \in \mathbf{C} \in \mathrm{I}\left(\overline{\mathbb{Z}}_{\max } \llbracket \gamma \rrbracket\right)^{q \times n}$, each entry of matrices $\mathbf{A}, \mathbf{B}, \mathbf{C}$ are intervals with bounds in dioid $\overline{\mathbb{Z}}_{\max } \llbracket \gamma \rrbracket$ with only non-negative exponents and coefficients integer values. By Theorem 1, Equation (9) has the minimum solution $X=A^{*} B U$. Therefore, $Y=C A^{*} B U$ and the transfer function of the system is $H=C A^{*} B \in \mathbf{H}=\mathbf{C A}^{*} \mathbf{B} \in \mathrm{I}\left(\overline{\mathbb{Z}}_{\max } \llbracket \gamma \rrbracket\right)^{q \times p}$, where $\mathbf{H}$ represents the interval in which the transfer function will be lie for all the variations of the parameters .

Figure 1 shows a TEG with 2 inputs and 1 output, which may represent a manufacturing system with 3 machines. Machines $M_{1}$ and $M_{2}$ produce parts assembled on machine $M_{3}$. A token in dotted lines means that the resource can or not to be available to manufacture part. Durations in bracket gives the minimal and maximal time spent in the place before contributing to the enabling of the downstream transition.

For instance, machine $M_{2}$ can manufacture 2 or 3 parts and each processing time will last 3 time units. Each manufactured

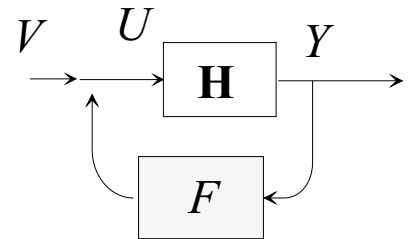

Fig. 2. An uncertain system with a feedback controller

part will spend between 2 and 6 time units in the downstream place before contributing to the enabling of transition $x_{3}$. Entries $\mathbf{A}_{2,2}=\left[3 \gamma^{3}, 3 \gamma^{2}\right]$ and $\mathbf{A}_{3,2}=[2,6]$ describe the intervals in which these parameters evolve. Therefore, we obtain the following interval matrices,

$$
\begin{aligned}
\mathbf{A} & =\left(\begin{array}{ccc}
{\left[2 \gamma^{2}, 5 \gamma\right]} & {[\varepsilon, \varepsilon]} & {[\varepsilon, \varepsilon]} \\
{[\varepsilon, \varepsilon]} & {\left[3 \gamma^{3}, 3 \gamma^{2}\right]} & {[\varepsilon, \varepsilon]} \\
{[3 \gamma, 4 \gamma]} & {[2,6]} & {\left[2 \gamma^{3}, 3 \gamma\right]}
\end{array}\right) \\
\mathbf{B} & =\left(\begin{array}{cc}
{[e, e]} & {[\varepsilon, \varepsilon]} \\
{[\varepsilon, \varepsilon]} & {[e, e]} \\
{[\varepsilon, \varepsilon]} & {[\varepsilon, \varepsilon]}
\end{array}\right) \\
\mathbf{C} & =\left(\begin{array}{lll}
{[\varepsilon, \varepsilon]} & {[\varepsilon, \varepsilon]} & [e, e]) .
\end{array}\right.
\end{aligned}
$$

and thanks to theorem 3, the transfer function $H$ belongs to the interval matrix $\mathbf{H}$ given below. It characterizes the whole transfer functions coming from (11):

$$
\mathbf{H}=\mathbf{C A}^{*} \mathbf{B}=\left(\left[3 \gamma\left(2 \gamma^{2}\right)^{*}, 4 \gamma(5 \gamma)^{*}\right]\left[2\left(3 \gamma^{3}\right)^{*}, 6(3 \gamma)^{*}\right]\right)
$$

\section{ROBUST FEEDBACK CONTROLLER SYNTHESIS}

We consider the behavior of a $p$-input $q$-output TEG by a state representation such as (9) and (10), we focus here on output feedback controller synthesis denoted $F$, added between the output $Y$ and the input $U$ of the system (see Figure 2). Therefore the process input verifies $U=V \oplus F Y$, and the output is described by $Y=H(V \oplus F Y)$. According to Theorem 1, the closed-loop transfer relation (depending on $F$ ) is then equal to

$$
Y=(H F)^{*} H V .
$$

where $H \in \mathbf{H}$ is the uncertain system transfer.

The objective of the robust feedback synthesis is to compute a controller $F$ which imposes a desired behavior (a specification) to the uncertain system. The problem addressed here consists in computing the greatest interval (in the sense of the order relation $\left.\preceq_{\mathrm{I}\left(\overline{\mathbb{Z}}_{\max } \llbracket \gamma \rrbracket\right)}\right)$, denoted $\hat{\mathbf{F}}$, which guarantees that the behavior of the closed loop system is lower than $\mathbf{G}_{r e f} \in \mathrm{I}\left(\overline{\mathbb{Z}}_{\max } \llbracket \gamma \rrbracket\right)^{q \times p}$ (a specification defined as an interval) for all $H \in \mathbf{H}$. Formally the problem consists in computing the upper bound of the following set

$$
\left\{\mathbf{F} \in \mathbf{I}\left(\overline{\mathbb{Z}}_{\max } \llbracket \gamma \rrbracket\right)^{p \times q} \mid(\mathbf{H F})^{*} \mathbf{H} \preceq \mathbf{G}_{r e f}\right\}
$$

Proposition 2 shows that this problem admits a solution for some reference models. 
Proposition 2: Let $\quad M_{\mathbf{H}} \quad$ : $\quad \mathrm{I}\left(\overline{\mathbb{Z}}_{\max } \llbracket \gamma \rrbracket\right)^{p \times q} \quad \rightarrow$ $\mathrm{I}\left(\overline{\mathbb{Z}}_{\max } \llbracket \gamma \rrbracket\right)^{q \times p}, \mathbf{F} \mapsto(\mathbf{H F})^{*} \mathbf{H}$ be a mapping. Let us consider the following sets :

$$
\begin{aligned}
& \mathbf{G}_{1}=\left\{\mathbf{G} \in \mathrm{I}\left(\overline{\mathbb{Z}}_{\max } \llbracket \gamma \rrbracket\right)^{q \times p} \mid \exists \mathbf{D} \in \mathrm{I}\left(\overline{\mathbb{Z}}_{\max } \llbracket \gamma \rrbracket\right)^{q \times q} \mathrm{~s}\right. \\
& \mathbf{G}_{2}=\left\{\mathbf{G} \in \mathrm{I}\left(\overline{\mathbb{Z}}_{\max } \llbracket \gamma \rrbracket\right)^{q \times p} \mid \exists \mathbf{D} \in \mathrm{I}\left(\overline{\mathbb{Z}}_{\max } \llbracket \gamma \rrbracket\right)^{p \times p}\right. \text { s.t. }
\end{aligned}
$$

If $\mathbf{G}_{\text {ref }} \in \mathbf{G}_{1} \cup \mathbf{G}_{2}$, there exists a greatest $\mathbf{F}$ such that $M_{\mathbf{H}}(\mathbf{F}) \preceq \mathbf{G}_{r e f}$, given by

$$
\hat{\mathbf{F}}=\underset{\left\{\mathbf{F} \in \mathrm{I}\left(\overline{\mathbb{Z}}_{\max } \llbracket \gamma \rrbracket\right)^{p \times q} \mid(\mathbf{H F})^{*} \mathbf{H} \preceq \mathbf{G}_{r e f}\right\}}{\bigoplus_{p}}
$$

Proof: First $\mathbf{G}_{r e f} \in \mathbf{G}_{1} \Leftrightarrow \exists \mathbf{D}$ s.t. $\mathbf{G}_{r e f}=\mathbf{D}^{*} \mathbf{H}$, then we seek the greatest solution to $(\mathbf{H F})^{*} \mathbf{H} \preceq \mathbf{D}^{*} \mathbf{H}$ which may be written as $(\mathbf{e} \bar{\oplus} \mathbf{H F} \bar{\oplus} \mathbf{H F H F} \bar{\oplus} \ldots) \mathbf{H} \preceq \mathbf{D}^{*} \mathbf{H}$, then the greatest inequality must satisfy the set of the following inequalities :

$$
\begin{aligned}
\mathbf{H} & \preceq \mathbf{D}^{*} \mathbf{H} \\
\mathbf{H F H} & \preceq \mathbf{D}^{*} \mathbf{H} \\
\mathbf{H F H F H} & \preceq \mathbf{D}^{*} \mathbf{H} \\
& \ldots \quad \text { etc }
\end{aligned}
$$

Kleene star definition gives $\mathbf{D}^{*} \succeq$ e (see remark (2)), thus inequality (16) is obviously satisfied. Thanks to proposition 1 , the greatest solution to inequality (17) is $\hat{\mathbf{F}}=\mathbf{H} \phi \mathbf{D}^{*} \mathbf{H} \phi \mathbf{H}$. Furthermore, $\hat{\mathbf{F}}$ is also a solution of inequality (18), indeed by recalling that $\mathbf{D}^{*} \mathbf{D}^{*}=\mathbf{D}^{*}$ and since $\mathbf{H} \hat{\mathbf{F}} \mathbf{H} \preceq \mathbf{D}^{*} \mathbf{H}$ we obtain

\section{$\mathbf{H F} H \hat{F} \mathbf{H} \preceq D^{*} \mathbf{H} \hat{F} \mathbf{H} \preceq D^{*} D^{*} H=D^{*} H$}

The same holds true recursively for the next inequalities, then $\hat{\mathbf{F}}$ is the greatest solution. We would show that $\hat{\mathbf{F}}$ is also the greatest solution if $\mathbf{G}_{r e f} \in \mathbf{G}_{2}$ with analog steps.

Corollary 2: If $\mathbf{G}_{\text {ref }} \in \operatorname{Im} M_{\mathbf{H}}$, then $\hat{\mathbf{F}}=\mathbf{H} \phi \mathbf{G}_{\text {ref }} \phi \mathbf{H}$ is the greatest solution to the equation $(\mathbf{H F})^{*} \mathbf{H}=\mathbf{G}_{\text {ref }}$.

Proof: First $\operatorname{Im} M_{\mathbf{H}} \subseteq\left(\mathbf{G}_{1} \cap \mathbf{G}_{2}\right)$, thus $\hat{\mathbf{F}}$ is the greatest $\mathbf{F}$ such that $M_{\mathbf{H}}(\mathbf{F}) \preceq \mathbf{G}_{r e f}$. Furthermore, $\forall \mathbf{y} \in \operatorname{Im} M_{\mathbf{H}}$, $M_{\mathbf{H}}(\mathbf{x})=\mathbf{y}$ admits a solution then $\hat{\mathbf{F}}$ is the greatest (see Property 1).

Below, we consider the robust controllers set, denoted $\mathcal{F}$, such that the transfer of the closed loop system is in $\mathbf{G}_{r e f}$ for all $H \in \mathbf{H}$

$$
\mathcal{F}=\left\{F \in \overline{\mathbb{Z}}_{\max } \llbracket \gamma \rrbracket^{p \times q} \mid(\mathbf{H} F)^{*} \mathbf{H} \subset \mathbf{G}_{r e f}\right\}
$$

Corollary 3: If $\mathbf{G}_{\text {ref }} \in \operatorname{Im} M_{\mathbf{H}}$, then $\hat{\mathbf{F}} \subset \mathcal{F}$.

Proof: If $\mathbf{G}_{r e f} \in \operatorname{Im} M_{\mathbf{H}}$, then $M_{\mathbf{H}}(\hat{\mathbf{F}})=\mathbf{G}_{r e f}$ thanks to Corollary 2, thus $(\mathbf{H} \hat{\mathbf{F}})^{*} \mathbf{H} \subset \mathbf{G}_{r e f}$. Obviously, this is equivalent to $\forall F \in \hat{\mathbf{F}},(\mathbf{H} F)^{*} \mathbf{H} \subset \mathbf{G}_{r e f}$, which leads to the result.

Corollary 3 shows that if $\mathbf{G}_{\text {ref }} \in \operatorname{Im} M_{\mathbf{H}}$ each feedback controller $F \in \hat{\mathbf{F}}$ is also in $\mathcal{F}$. From a practical point of view this means that for all number of tokens and holding time belonging to the given interval the closed loop system will be in the specification interval.
Remark 4: From a computational point of view, we have

$$
\begin{aligned}
\hat{\mathbf{F}} & =\mathbf{H} \phi \mathbf{G}_{r e f} \phi \mathbf{H}=[\underline{H}, \bar{H}] \phi\left[\underline{G}_{r e f}, \bar{G}_{r e f}\right] \phi[\underline{H}, \bar{H}]=\left[\underline{H} \phi \underline{G}_{r e f} \wedge \bar{H} \phi \bar{G}_{r e f}, \overline{]}\right. \\
& =\left[\left(\underline{H} \phi \underline{G}_{r e f} \wedge \bar{H} \phi \bar{G}_{r e f}\right) \phi \underline{H} \wedge \bar{H} \phi \bar{G}_{r e f} \phi \bar{H}, \bar{H} \phi \bar{G}_{r e f} \phi \bar{H}\right]
\end{aligned}
$$

t. $\mathbf{G}=\underline{\mathbf{D}}^{*} \mathbf{H}\left[\underline{I} \phi \underline{G}_{r e f} \phi \underline{H} \wedge \bar{H} \phi \bar{G}_{r e f} \phi \underline{H} \wedge \bar{H} \phi \bar{G}_{r e f} \phi \bar{H}, \bar{H} \phi \bar{G}_{r e f} \phi \bar{H}\right]$

thanks

$\left.\mathbf{G}=\mathbf{H D}^{*}\right\}$.

The last equation may be simplified, indeed $\left(\bar{H} \phi \bar{G}_{r e f}\right) \phi \underline{H} \succeq$

$\left(\bar{H} \phi \bar{G}_{r e f}\right) \phi \bar{H}$ thanks to the antitony of mapping $a \phi x$ (i.e., $\left.x_{1} \succeq x_{2} \Rightarrow a \phi x_{1} \preceq a \phi x_{2}\right)$, then $\bar{H} \phi \bar{G}_{r e f} \phi \underline{H} \wedge \bar{H} \phi \bar{G}_{r e f} \phi \bar{H}=$ $\bar{H} \phi \bar{G}_{r e f} \phi \bar{H}$. Therefore

$$
\hat{\mathbf{F}}=\mathbf{H} \phi \mathbf{G}_{r e f} \phi \mathbf{H}=\left[\underline{H} \phi \underline{G}_{r e f} \phi \underline{H} \wedge \bar{H} \phi \bar{G}_{r e f} \phi \bar{H}, \bar{H} \phi \bar{G}_{r e f} \phi \bar{H}\right] .
$$

\section{EXAMPLE : OUtPUt FEEDBACK SYNTHESIS}

We describe a complete synthesis of a controller for the uncertain TEG depicted with solid black lines in Fig. 1. The reference model chosen is

$$
\begin{aligned}
G_{r e f} & =\left(\mathbf{H}\left(\begin{array}{l}
\gamma^{2} \\
\gamma^{2}
\end{array}\right)\right)^{*} \mathbf{H} \\
& =\left([ 3 \gamma \oplus 5 \gamma ^ { 3 } ( 1 \gamma ) ^ { * } , 4 \gamma ( 5 \gamma ) ^ { * } ] \quad \left[2 \oplus\left(4 \gamma^{2}\right)(1 \gamma)^{*}, 6 \oplus 9 \gamma \oplus 12 \gamma^{2} \oplus 15 \gamma^{3} \oplus 18 \gamma^{4} \oplus 2\right.\right.
\end{aligned}
$$

This specification means that not more than two tokens can input in the TEG at the same moment. We refer the reader to [6] for a discussion about reference model choice. We aim to compute the greatest interval of robust controllers which keep the same objective.

According to Proposition 2 and solution (15), the controller is obtained by computing $\mathbf{H} \nmid \mathbf{G}_{r e f} \phi \mathbf{H}$. Therefore we obtain

$$
\hat{\mathbf{F}}=\left(\begin{array}{c}
{\left[-3 \gamma^{-1} \oplus-1 \gamma(1 \gamma)^{*},-15 \gamma^{-1}(5 \gamma)^{*}\right]} \\
{\left[-2 \oplus \gamma^{2}(1 \gamma)^{*},-6 \oplus-3 \gamma \oplus \gamma^{2} \oplus 3 \gamma^{3} \oplus 6 \gamma^{4} \oplus 9 \gamma^{5} \oplus 13 \gamma^{6}(5 \gamma)^{*}\right]}
\end{array}\right)
$$

For the realization of that controller it is necessary to choose one feedback in the set $\hat{\mathbf{F}}$. Here we choose the lower bound of this set, i.e.,

$$
\underline{\hat{F}}=\left(-3 \gamma^{-1} \oplus-1 \gamma(1 \gamma)^{*} \quad-2 \oplus \gamma^{2}(1 \gamma)^{*}\right)^{t}
$$

This feedback is not causal because there are negative coefficients in matrix entries meaning negative date for the transition firings (see [2] for a strict definition of causality in dioid). The canonical injection from the set of causal elements of $\overline{\mathbb{Z}}_{\max } \llbracket \gamma \rrbracket$ (denoted $\overline{\mathbb{Z}}_{\max }^{+} \llbracket \gamma \rrbracket$ ) in $\overline{\mathbb{Z}}_{\max } \llbracket \gamma \rrbracket$ is also residuated (see [6] for details). Its residual is denoted $\mathrm{Pr}_{+}$, therefore the greatest causal feedback is

$$
\underline{\hat{F}}_{+}=\operatorname{Pr}_{+}(\underline{\hat{F}})=\left(\begin{array}{c}
\gamma^{2}(1 \gamma)^{*} \\
\gamma^{2}(1 \gamma)^{*}
\end{array}\right)
$$

Figure 1 shows one realization of the controller (bold dotted lines). Figure 3 shows the interval of transfer in which the real transfer is assumed evolving, and the interval of the specification in which the closed loop system will evolve in a guaranteed way.

Remark 5: The reader can find software tools in order to handle periodic series and solve the illustration (see [12]). 


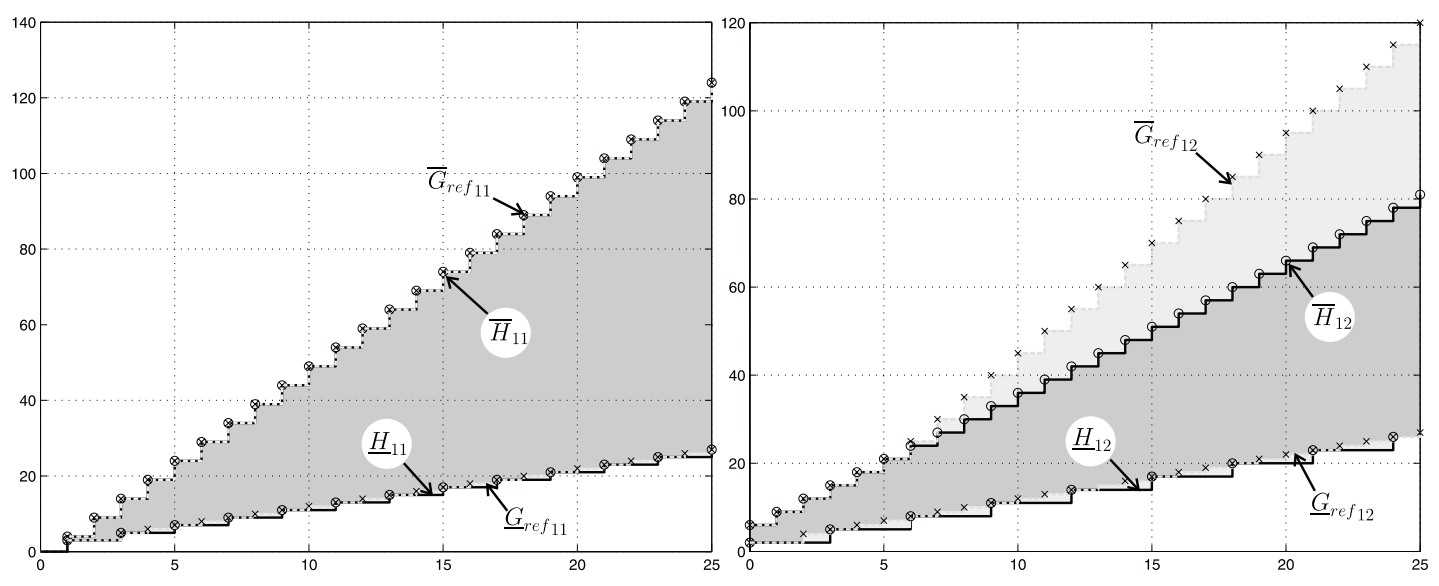

Fig. 3. The interval of transfer relation of the uncertain system (two entries $\left[\underline{H}_{11}, \bar{H}_{11}\right]$ and $\left.\left[\underline{H}_{12}, \bar{H}_{12}\right]\right)$, and the interval of the specification $\left(\left[\underline{G}_{r e f_{11}}, \bar{G}_{r e f_{11}}\right]\right.$ and $\left.\left[\underline{G}_{r e f_{12}}, \bar{G}_{r e f_{12}}\right]\right)$ equal to the interval in which the closed loop system will evolve.

\section{CONCLUSION}

In this paper we have assumed that the TEG includes some parametric uncertainties in a bounded context. We have given a robust feedback controller synthesis which ensures that the closed-loop system transfer is in a given interval for all feasible values for the parameters. The next step is to extend this work to other control structure such as the one given in [11]. The traditional interval theory is very effective for parameter estimation, it would be interesting to apply the results of this paper to the TEG parameter estimation such as intended in [7].

\section{REFERENCES}

[1] H. Ayhan and M.A. Wortman. Job flow control in assembly operations. IEEE Transactions on Automatic Control, 44(4):864-868, 1999.

[2] F. Baccelli, G. Cohen, G.J. Olsder, and J.P. Quadrat. Synchronization and Linearity: An Algebra for Discrete Event Systems. Wiley and Sons, 1992.

[3] T.S. Blyth and M.F. Janowitz. Residuation Theory. Pergamon press, 1972.

[4] J.-Y Le Boudec and P. Thiran. Network Calculus. Springer Verlag, 2002.

[5] G. Cohen, P. Moller, J.P. Quadrat, and M. Viot. Algebraic Tools for the Performance Evaluation of Discrete Event Systems. IEEE Proceedings: Special issue on Discrete Event Systems, 77(1):39-58, January 1989.

[6] B. Cottenceau, L. Hardouin, J.-L. Boimond, and J.-L. Ferrier. Model Reference Control for Timed Event Graphs in Dioid. Automatica, 37:1451-1458, August 2001.

[7] L. Jaulin, J.-L. Boimond, and L. Hardouin. Estimation of discrete event systems using interval computation. Reliable Computing, 5:165-173, 1999.

[8] L. Jaulin, M. Kieffer, O. Didrit, and E. Walter. Applied Interval Analysis with Examples in Parameter and State Estimation, Robust Control and Robotics. Springer-Verlag, London, 2001.

[9] G.-L. Litvinov and A.-N. Sobolevskii. Idempotent Interval Analysis and Optimization Problems. Reliable Computing, 7(5), 2001.

[10] R. Lüders and R. Santos-Mendes. Generalized Multivariable Control of Discrete Event Systems in Dioid. Worshop on Discrete Event Systems, WODES'02, Zaragoza, 2002.

[11] C.-A. Maia, L. Hardouin, R. Santos-Mendes, and B. Cottenceau. Optimal closed-loop control of timed-event graphs in dioids. To appear in IEEE Transactions on Automatic Control.

[12] SW2001. Software Tools for Manipulating Periodic Series. http://www.istia-angers.fr/hardouin/outils.html, http://amadeus.inria.fr/gaubert/papers/max.html., 2001. 